\title{
A Hybrid Stochastically Perturbed Parametrization Scheme in a Convection-Permitting Ensemble
}

\author{
Clemens Wastl, Yong Wang, Aitor Atencia, And Christoph Wittmann \\ Department of Forecasting Models, Zentralanstalt für Meteorologie und Geodynamik, Vienna, Austria
}

(Manuscript received 29 November 2018, in final form 4 March 2019)

\begin{abstract}
Model error in ensemble prediction systems is often represented by either a tendency perturbation approach or a process-based parameter perturbation scheme. In this paper a novel hybrid stochastically perturbed parameterization (HSPP) scheme is proposed and implemented in the Convection Permitting Limited Area Ensemble Forecasting (C-LAEF) system developed at the Zentralanstalt für Meteorologie und Geodynamik (ZAMG). In HSPP, the individual parameterization tendencies of the physical processes radiation, shallow convection, and microphysics are perturbed stochastically by a spatially and temporally varying pattern. Uncertainties in the turbulence scheme are considered by perturbing key parameters on the process level. The proposed scheme HSPP features several advantages compared to the popular stochastically perturbed parameterization tendencies (SPPT) scheme: it considers a more physically consistent relationship between different parameterization schemes, deals with uncertainties especially adapted to the individual physical processes, respects conservation laws of energy and moisture, and eliminates the tapering function that has to be introduced to the SPPT scheme because of mainly numerical reasons. The hybrid scheme HSPP is evaluated over one summer and one winter month and compared to a reference ensemble without any stochastic physics perturbations and to two versions of the SPPT scheme. The results show that HSPP significantly increases the ensemble spread of temperature, humidity, wind speed, and pressure, especially in the lower levels of the atmosphere where a tapering function is active in the original SPPT approach. Precipitation verification yields a generally improved probabilistic performance of the HSPP scheme in summer when convection is dominating, which has also been demonstrated in a case study.
\end{abstract}

\section{Introduction}

Despite the fact that many regional numerical weather prediction (NWP) models resolve individual convective cells, they still include a lot of uncertainties that have to be considered. There are uncertainties originating from the insufficient representation of the present state of the atmosphere (initial condition errors), uncertainties coming from the lateral boundary conditions [in case of limited-area models (LAMs)], and inaccuracies in the model itself (model error-computational constraints, parameterizations, etc.). Because of these deficiencies the current trend at many meteorological services [e.g., European Centre for Medium-Range Weather Forecasts (ECMWF)] is to move away from single deterministic forecasts toward probabilistic products.

The representation of model error in an ensemble prediction system can be achieved in different ways: (i) multimodel approach where the output of different

\footnotetext{
Corresponding author: Yong Wang, yong.wang@zamg.ac.at
}

NWP models is combined (utilized in some LAMs; Iversen et al. 2011); (ii) multiphysics approach where different physics parameterizations are used in one single numerical model (Charron et al. 2010; Wang et al. 2011); (iii) random parameters (RP) approach where some of the key parameters in the physical parameterizations are varied within an ensemble (Baker et al. 2014; Bowler et al. 2008); and (iv) the use of stochastic physics (Palmer et al. 2009; Berner et al. 2009). In the latter approach each member of an ensemble receives a different but equally likely stochastic forcing usually generated by a pattern generator. This method is becoming more used since it is computationally inexpensive and has shown to significantly improve the reliability of weather forecasts (Leutbecher et al. 2017). Within stochastic physics there exist several methods: 1) stochastic kinetic energy backscatter scheme (SKEB; Berner et al. 2009), which considers interactions between the different scales in a numerical model; 2) perturbation of parameterized model tendencies (Palmer et al. 2009); and 3) physically motivated methods that address sources of uncertainty 
in a particular parameterization scheme (Plant and Craig 2008; Bengtsson et al. 2013; Kober and Craig 2016). A widely used stochastic physics scheme is the stochastically perturbed parameterization tendencies (SPPT), which has been developed at the ECMWF (Buizza et al. 1999; refined by Palmer et al. 2009). In SPPT a spectral pattern generator produces temporal and spatial consistent noise which is applied to the net model tendencies of temperature, wind speed and humidity coming from the physics parameterizations. In the Integrated Forecast System (IFS) implementation (Palmer et al. 2009), three independent random patterns with different correlation scales are used to span the uncertainty at the mesoscale, synoptic, and planetary scale. SPPT is simple and effective in improving the ensemble forecast skill of many global and regional ensemble systems (e.g., Charron et al. 2010; Bouttier et al. 2012; Romine et al. 2014; Weisheimer et al. 2014). However, SPPT has also been criticized for its lack of physical consistency (Leutbecher et al. 2017; Ollinaho et al. 2017).

First of all, SPPT does not respect conservation laws (e.g., energy) because only net physics tendencies in the atmosphere are perturbed without considering fluxes at the surface or top of the atmosphere. This energy imbalance can cause numerical instabilities and therefore a tapering function, which reduces the perturbations smoothly to zero in the boundary layer and in the stratosphere, is required in some NWP models as in the IFS (Leutbecher et al. 2017) or Applications of Research to Operations at Mesoscale (AROME; Bouttier et al. 2012) ensemble. On the other hand, this tapering function implies a reduced model error in the lowest and topmost parts of the atmosphere, which is unrealistic.

Because of its multiplicative characteristics, SPPT does not affect areas where the net tendency of a parameter is zero, even though the partial tendencies of the individual parameterizations might be large. This inconsistency has been reconsidered by Christensen et al. (2017) in the iSPPT (independent SPPT) approach where each parameterization receives its own adapted noise. iSPPT is physically more consistent but still reveals the drawback of not fully respecting the energy conservation.

Another possibility to represent the model uncertainty directly at the process level is to perturb poorly constrained parameters in the physics parameterizations. This has been implemented for example in the Met Office Global and Regional Ensemble Prediction System (MOGREPS; Bowler et al. 2008). Further developments of this random parameter scheme have been suggested by Hacker et al. (2011) and Christensen et al. (2015). Recently, Ollinaho et al. (2017) proposed the stochastically perturbed parameterizations (SPP) scheme for the IFS model. In SPP, spatially and temporally varying perturbations are applied to 20 preselected parameters in the IFS parameterizations of turbulent diffusion, subgrid orography, convection, clouds, large-scale precipitation, and radiation. In this way model uncertainty is directly represented in the parameterizations itself and can be addressed to processes that have a big uncertainty and are known to contribute significantly to the skill of the forecast. The advantage of SPP is that the local budgets of energy and moisture are conserved and that the physically consistent relationship between the different physics variables is kept. Furthermore, SPP generates realistic perturbations in the boundary layer and at the top of the atmosphere as it does not need the pragmatic tapering function (Leutbecher et al. 2017). A comprehensive verification of SPP has shown a good potential of this process-based approach for some surface parameters in the first hours, but for most upper-air variables at later lead times SPPT performs better (Ollinaho et al. 2017). Furthermore, SPP is a complex scheme that demands much more resources to maintain than the simple SPPT scheme. SPP has also shown good potential at the convection-permitting scale, which has been demonstrated by Jankov and Beck (2019) who implemented it to the High-Resolution Rapid Refresh update cycle land surface model (HRRR). They conclude that SPP has a positive impact on the skill and reliability of some surface meteorological parameters like the low-level wind forecasts.

Both schemes, SPPT and SPP have their strengths and weaknesses and therefore it is plausible to combine both schemes into a hybrid system. Following this, we propose in the present paper a novel hybrid stochastically perturbed parameterization scheme (HSPP) for addressing the model error in an ensemble forecasting system. The idea is to quantify the model uncertainties at a process level by combining tendency perturbations (SPPT) and parameter perturbations (SPP). The system should on the one hand keep the advantages of SPPT such as simplicity and effectiveness, but should on the other hand represent the physical consistent relationship of SPP and thus avoid numerical instabilities in the boundary layer as in SPPT.

In the proposed HSPP we apply the physical parameterization-based SPPT approach (pSPPT; Wastl et al. 2019, hereafter W19) to deal with uncertainties in the radiation, shallow convection and microphysics scheme of the Convection Permitting Limited Area Ensemble Forecasting (C-LAEF) system. In pSPPT the partial tendencies of the different parameterization schemes receive their own adapted noise, instead of 
perturbing the total net tendencies as in SPPT. Uncertainties in the turbulence scheme of HSPP are considered by applying perturbations directly on key parameters at the process level, as in SPP. The turbulence scheme has been chosen for a parameter perturbation approach, because it is mainly responsible for the energy exchange between surface and atmosphere and is vulnerable to numerical instabilities when applying a tendency perturbation approach (W19).

In the present paper we give a detailed description of the HSPP methodology in section 2; we investigate the performance of HSPP, SPPT and pSPPT in comparison to an ensemble without stochastic physics (REF) in section 3; and in section 4 we discuss various aspects of the proposed HSPP system.

\section{Methodology}

\section{a. The C-LAEF system}

The C-LAEF system is currently under development at the Austrian weather service Zentralanstalt für Meteorologie und Geodynamik (ZAMG). It is an ensemble prediction system based on the convection-permitting model AROME (Seity et al. 2011). AROME is under active development within the Aire Limitée Adaptation Dynamique Développement International (ALADIN; Termonia et al. 2018), High Resolution Limited Area Model (HIRLAM; Bengtsson et al. 2017), and Regional Cooperation for Limited Area Modeling in Central Europe (RC LACE; Wang et al. 2018) consortia. The model is operated on a $2.5-\mathrm{km}$ grid centered on Austria and covers the Alpine region (Fig. 1) with 90 vertical levels and a time step length of $60 \mathrm{~s}$. The forecast range is $30 \mathrm{~h}$ with an output frequency of $1 \mathrm{~h}$. The AROME model has a nonhydrostatic dynamical kernel (Bénard et al. 2010) and uses a physics package developed for the Meso-NH model (Mascart and Bougeault 2011).

C-LAEF comprises 16 members that are coupled with the first 16 members out of a total of 51 from the global ensemble system of the ECMWF. A Davies relaxation scheme (Davies 1976) is used for coupling with a coupling frequency of $3 \mathrm{~h}$. For the present study C-LAEF runs in pure downscaling mode without any initial or surface perturbations and no data assimilation. This is deemed acceptable since we are only interested in a relative comparison of different methods of stochastic physics. In all the experiments with stochastic physics presented here, the perturbations are produced by a spectral pattern generator that has been developed at the ECMWF (Palmer et al. 2009) and transformed to be used in the LAM model AROME by Bouttier et al. (2012).

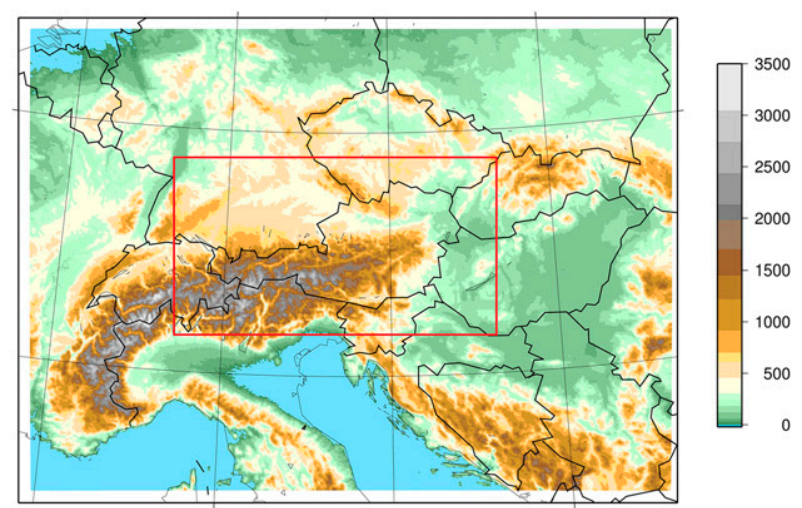

FIG. 1. Domain of the C-LAEF system including the INCA domain for precipitation verification (red box). The color shows the altitude (m).

\section{b. A hybrid stochastically perturbed parameterization scheme (HSPP)}

Stochastic physics schemes perturbing model tendencies (SPPT, iSPPT, pSPPT) and process-based parameter perturbation schemes like SPP address quite different mechanisms for the representation of model uncertainty. The perturbation of different schemes by different methodologies allows the combination of them into a complete hybrid system (HSPP). In this hybrid HSPP system, the pSPPT (W19) approach is applied to the radiation, shallow convection and microphysics parameterization scheme of C-LAEF, while uncertainty in turbulence is introduced by perturbing some key parameters in the turbulence scheme as in SPP. Combining pSPPT and SPP into one hybrid system enables the tapering function to be removed completely without any consequences on the model stability.

\section{1) TENDENCY PERTURBATION IN HSPP}

The different physics schemes (radiation, shallow convection, turbulence and microphysics) in C-LAEF are called subsequently and each scheme provides a partial tendency of the main model quantities $(T, U$, $V, Q)$. The condensed water species are adjusted at each time step by the fast microphysics step (Seity et al. 2011).

In the pSPPT approach (W19) the partial tendencies of the physics parameterization schemes are perturbed separately and directly after the call of each parameterization. Interactions between the individual physics schemes are possible in pSPPT by considering the perturbed fields of one physics scheme in the subsequent one. In other words, the uncertainties are passed through the different schemes and can be enhanced or diminished by the following ones. The iSPPT approach developed by Christensen et al. (2017) and applied to the IFS is very similar, but interactions 

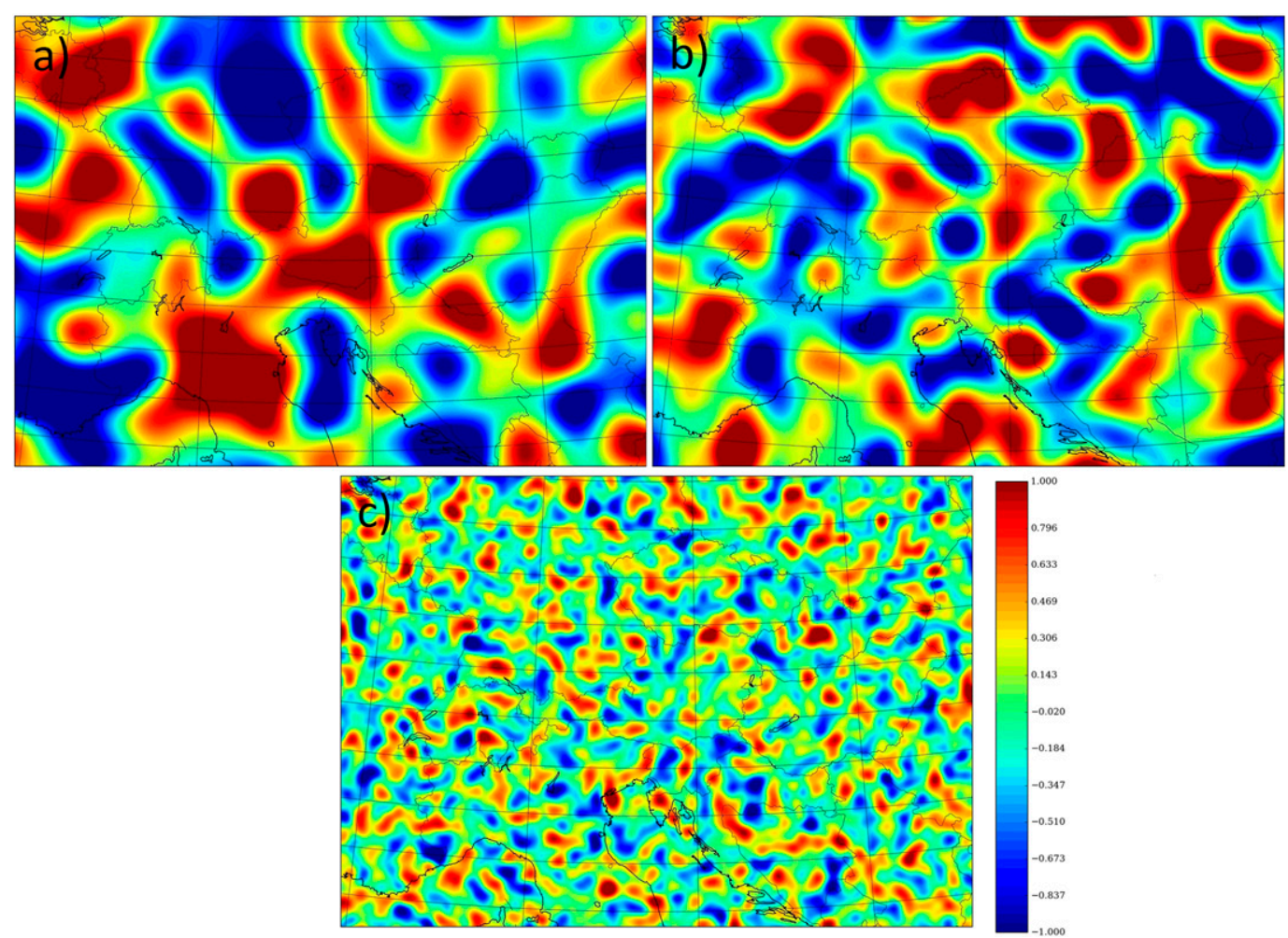

FIG. 2. Perturbation fields (domain of Fig. 1) applied to the different physics parameterization schemes in the pSPPT and HSPP approach. (a) Radiation, (b) shallow convection, and (c) microphysics. The characteristics of the perturbation fields can be found in Table 1 .

between the schemes are not possible in this method because the perturbations are applied at the end of each time step. The W19 study revealed several deficiencies in the pSPPT approach that have been eliminated in the present updated version. First of all, the very simple supersaturation adjustment is replaced. In the original version [introduced by Bouttier et al. (2012)] stochastic perturbations are not applied if the saturation level is reached or if the following humidity falls below zero. Supersaturation can only be reached due to positive humidity or negative temperature perturbations-omitting perturbations in such cases therefore results in a general trend toward a systematic drying and warming of the atmosphere. This effect was already highlighted by several SPPT studies (Berner et al. 2009; Bouttier et al. 2012; W19). The updated supersaturation check (Szúcs 2016) is an iterative method where the first step defines the maximum possible perturbation of humidity and temperature at each grid point. In case of a possible supersaturation, only this reduced perturbation is applied and additionally the opposite part of the Gaussian distributed perturbation field is also reduced, respectively. With this method the distribution remains symmetric and humidity is conserved.

Second, the structure of the stochastic noise in the updated version of PSPPT is adapted separately to the individual physics parameterizations. In W19 the perturbation fields of radiation, shallow convection, turbulence, and microphysics differed only in the seed. Now the pattern generator is run separately for each parameterization scheme with adjusted horizontal and temporal structures of the noise. In this way different uncertainty scales of the parameterizations are considered. For example, perturbations applied to the shallow convection scheme are of a much larger scale than for the microphysics scheme (Fig. 2). The parameter values controlling the different stochastic perturbation fields can be found in Table 1. These individual settings have been found on personal assessment by running several tests to estimate approximately the uncertainty scale of each parameterization. The smallest-scale perturbations are applied to the scheme with the smallest uncertainty scale (microphysics).

The model stability of the current version of pSPPT has significantly increased compared to the original SPPT scheme applied to C-LAEF. As a consequence the very pragmatic tapering function can be switched off for all physics parameterizations except for turbulence. The imbalance between unperturbed surface fluxes and perturbed atmospheric tendencies creates too much instabilities in the turbulence scheme and 
TABLE 1. Parameter values for the stochastic fields used in the different parameterization schemes in C-LAEF.

\begin{tabular}{lccc}
\hline \hline $\begin{array}{c}\text { Parameterization } \\
\text { scheme }\end{array}$ & $\begin{array}{c}\text { Horizontal } \\
\text { correlation } \\
\text { length }(\mathrm{km})\end{array}$ & $\begin{array}{c}\text { Time } \\
\text { correlation } \\
\text { scale (s) }\end{array}$ & Std dev \\
\hline Radiation & 80 & 21600 & 0.5 \\
Shallow convection & 60 & 14400 & 0.5 \\
Microphysics & 30 & 7200 & 0.5 \\
\hline
\end{tabular}

therefore a reduction of perturbations (tapering) is needed there. Furthermore, turbulence processes are very sensitive and even small perturbations of tendencies near the ground can produce strong model instabilities. For these reasons, the tendency perturbation in the HSPP system is only applied to radiation, shallow convection, and microphysics schemes, but not to turbulence.

\section{2) PARAMETER PERTURbATION IN HSPP}

Uncertainty representation in the turbulence scheme of HSPP is done by a process-level approach. This method is based on the SPP scheme described in Ollinaho et al. (2017) where preselected parameters in the physics parameterizations are perturbed directly. The six parameters in the turbulence scheme that have been chosen are listed in Table 2. These parameters address processes such as dissipation of potential temperature and total kinetic energy or the turbulent mixing. These parameters are known to be uncertain and play a crucial role within the turbulence parameterization. Changing their values can have a significant impact on the forecast quality. Besides the variable names and the relevant processes in the turbulence scheme, Table 2 also shows the range of physically realistic values for each perturbed parameter and the standard deviation of the underlying Gaussian distribution of the perturbation field. The perturbed parameters $P i^{\prime}$ are obtained by multiplying the unperturbed parameters $(P i)$ by the exponential of the perturbation field $(\Psi i)$ :

$$
P i^{\prime}=\exp (\Psi i) P i .
$$

The unperturbed parameter is the value used in the deterministic forecast. A lognormal distribution has been chosen to retain the original sign of the unperturbed values without any need to adjust or clip the perturbation values. $\Psi i$ is produced by a spectral pattern generator that is run separately for each of the six selected parameters. All perturbation fields follow a Gaussian distribution with zero mean that implies that the median of the distribution of the perturbed parameter is equal to the unperturbed value. The stochastic pattern generator uses for all six parameters the same horizontal $(40 \mathrm{~km})$ and temporal $(2 \mathrm{~h})$ correlation scale, only the standard deviation of the Gaussian distribution is differing to obtain the appropriate range of the parameters in Table 2. Further details about this method can be found in Ollinaho et al. (2017).

\section{c. Experimentation and verification}

A set of four C-LAEF experiments (Table 3) has been chosen for a detailed verification over a longer period. To assess the quality of the four experiments in different weather/climate situations the verification is done for a summer (July 2016) and winter month (January 2017). Each C-LAEF experiment consists of 16 perturbed members and one run per day (0000 UTC) so that there are 31 ensemble forecasts in each 1-month period over which the scores are averaged. Surface verification is done for the variables sea level pressure, $2 \mathrm{~m}$ temperature and relative humidity, and $10 \mathrm{~m}$ wind speed by using SYNOP station data at more than 1200 observation sites in the investigation area. Precipitation verification is done on the grid by using the Integrated Nowcasting through Comprehensive Analysis (INCA; Haiden et al. 2011; Wang et al. 2017) analysis system developed at ZAMG. It combines rain gauge measurements and radar data by a sophisticated algorithm on a $1 \mathrm{~km}$ grid. For this purpose the precipitation fields of the C-LAEF experiments are interpolated to the INCA grid for a

TABLE 2. Parameters in the turbulence scheme of AROME physics that are perturbed in the hybrid system HSPP. The first column indicates the parameter name in the code, the second column specifies the physically realistic range of perturbed values, and the third column describes the relevant processes. The last column gives the standard deviation of the underlying Gaussian distribution of the perturbation field. BL89 stands for the Bougeault and Lacarrere (1989) scheme.

\begin{tabular}{lcll}
\hline \hline Parameter & Range & \multicolumn{1}{c}{ Description } \\
\hline XLINI & $0-0.1$ & Minimum BL89 mixing length & 0.5 \\
XCTD & $0.98-1.2$ & Constant for dissipation of potential temperature and mixing ratio \\
XCTP & $2.325-4.65$ & Constant for temperature-vapor pressure correlation & 0.05 \\
XCEP & $1.055-4.0$ & Constant for wind-pressure correlation & 0.2 \\
XCED & $0.7-0.85$ & Constant for dissipation of total kinetic energy (TKE) & 0.35 \\
XALPSBL & $3.75-4.65$ & Value related to the TKE universal function within the surface & 0.05 \\
& & boundary layer & 0.2 \\
\hline
\end{tabular}


TABLE 3. Name and description of the different experiments.

\begin{tabular}{lll}
\hline \hline Name & \multicolumn{1}{c}{ Perturbations } \\
\hline REF & No & Reference run without stochastic physics; downscaling of ECMWF ensemble \\
SPPT & Tendencies & Original SPPT perturbing total model tendencies; Palmer et al. (2009) \\
pSPPT & Tendencies & Perturbation of partial model tendencies; Wastl et al. (2019) \\
HSPP & Tendencies/parameters & Combination of pSPPT and parameter perturbation (SPP); detailed \\
& & description in section 2b \\
\hline
\end{tabular}

domain framing Austria (red rectangle in Fig. 1). The interpolation method selected is the bilinear interpolation but the results are not significantly different as the obtained by nearest neighbor. The verification of upperair variables like geopotential, temperature, specific humidity, and wind speed is done against ECMWF analyses at 13 levels between 1000 and $100 \mathrm{hPa}$. To assess the quality of the different C-LAEF experiments, standard probabilistic scores (spread, root-mean-square error, Brier score, continuous ranked probability score, etc.) are calculated for both, surface and upper-air variables. More details on the calculation of the scores are given in sections $3 \mathrm{~b}$ and $3 \mathrm{c}$. The statistical significance of the differences between the three experiments and the reference run is defined by using a bootstrapping confidence test. In this method, a block of 3 days is sampled out of the 31-day verification period (summer and winter, respectively) and the average score difference to the reference run is computed. This procedure is then repeated for 5000 times to gain an empirical distribution of all three experiments. Statistical significance is reached if the sign of the difference is not contradicted by more than $10 \%$ of the sample [for more details see Wilks (2011)].Observation errors are not taken into account in the verification because our investigation focuses on relative scores and we assume that the observation error would act in the same direction for all experiments.

\section{Results of the numerical experiments}

\section{a. Impact on upper air}

Figure 3 shows the upper-air verification results for all four experiments in the test periods July 2016 (Figs. 3a,c) and January 2017 (Figs. 3b,d). The scores are calculated by averaging over all grid points in the domain and over all 24-h-range forecasts in the respective period. Ensemble spread (solid lines) is given in absolute numbers while root-mean-square error (RMSE) is given as average difference between the experiments and the reference run REF [experiment (REF)]. Asterisks on the left (RMSE) and circles on the right (spread) indicate statistical significant differences. July 2016 was a typical Central European summer month with very unstable conditions and a lot of thunderstorm activity in the Alpine region causing local floods. Average ensemble spread of the $24 \mathrm{~h}$ temperature forecasts in this period lies between 0.5 and $0.7 \mathrm{~K}$ with the highest values near the surface and in the lower stratosphere around $200 \mathrm{hPa}$ (Fig. 3). Comparing the different experiments yields more or less equal values above $600 \mathrm{hPa}$ and relatively large differences in the lowest model levels. Near the surface the ensemble spread of pSPPT (red) and the hybrid system HSPP (green) is about $0.1 \mathrm{~K}$ higher than in the reference or original SPPT experiments. This increase is statistically significant and quite high considering a total spread of about $0.65 \mathrm{~K}$. The fact that the biggest differences are mainly restricted to the lowermost $400 \mathrm{hPa}$ of the atmosphere indicates the influence of the tapering function that reduces the perturbations in this layer in the SPPT experiment. The RMSE of the ensemble mean is generally very high in all C-LAEF experiments with values between $1.2 \mathrm{~K}$ near the surface and $0.6 \mathrm{~K}$ in the upper levels (not shown). This can be explained by not using any kind of data assimilation on the LAM side. The dashed lines in Fig. 3 reveal relatively small (and mostly not significant) differences between the experiments indicating a minor effect of stochastic physics on forecast error.

Calculating the spread-skill relationship (not shown) indicates that the C-LAEF system is mostly underdispersive implying too little spread compared to RMSE that also results in a reduced forecast reliability. The results of the specific humidity scores in July 2016 are a bit different. Ensemble spread is highest at about $850 \mathrm{hPa}$ and decreases rapidly above. The use of stochastic physics generally increases the ensemble spread in the levels up to $600 \mathrm{hPa}$, but there are hardly any differences between the three experiments with stochastic physics. The dashed lines indicate a slight decrease of RMSE of humidity in the lower levels for these three experiments. The simple supersaturation adjustment in SPPT causes a negative humidity bias in this experiment in most parts of the atmosphere (not shown). This has been fixed in PSPPT and HSPP by an improved supersaturation treatment. 

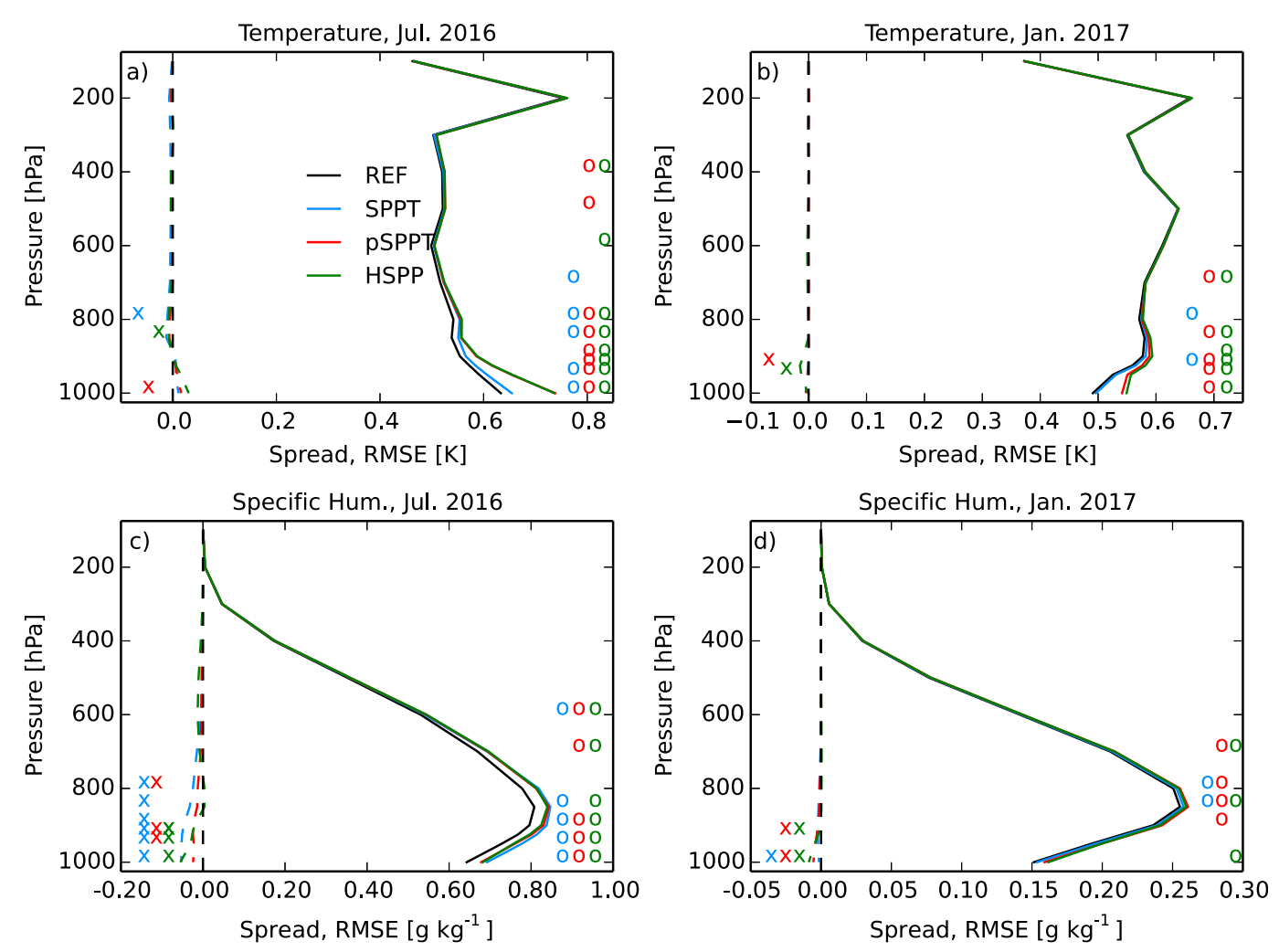

FIG. 3. Average vertical profiles of (a),(b) temperature and (c),(d) specific humidity for the 24-h-range forecasts in (a),(c) July 2016 and (b),(d) January 2017. The solid lines show the ensemble spread of all four experiments, and the dashed lines the RMSE difference to the reference run (REF). Asterisks on the left (RMSE) and circles on the right (spread) indicate statistically significant differences between the experiments and the reference.

The winter period January 2017 was characterized by very cold and stable conditions in the investigation area. The scores in the right column of Fig. 3 show a very similar RMSE of temperature in all four experiments while ensemble spread is significantly increased with stochastic physics below $800 \mathrm{hPa}$. The highest spread increase is generated with pSPPT and the hybrid system HSPP. Compared to summer, the influence on spread is generally smaller (about $0.05 \mathrm{~K}$ ) and restricted to lower levels, which can be explained by a much more stable stratification in the winter atmosphere (W19). In summer the radiative forcing, and accordingly also convection and turbulence, are much stronger and thus even small perturbations in the model can have a large effect. The generally small effect on the verification scores in winter is also obvious for humidity in the lower-right panel of Fig. 3.

\section{b. Impact on surface variables}

Average ensemble spread and RMSE scores for the surface parameters temperature, relative humidity, sea level pressure, and wind speed in July 2016 are shown in Fig. 4. Stochastic physics results in a significant increase in ensemble spread with the highest values for the pSPPT and HSPP runs. Spread increase compared to REF lies approximately in the range of $0.2 \mathrm{~K}$ for temperature, $1 \%$ for relative humidity, and $0.1 \mathrm{~m} \mathrm{~s}^{-1}$ for wind speed. The smallest effect of stochastic physics can be found for sea level pressure. The increasing spread also improves the forecast reliability in the experiments with stochastic physics (not shown).

For most parameters a distinctive diurnal cycle is evident in the spread scores with the highest values in the afternoon when strong convection is present in this season. The spread decreases immediately after sunset for all surface parameters, except for sea level pressure. The RMSE is shown here as a difference to REF because the large absolute forecast error would otherwise override the relatively small differences among the experiments in this figure. The absolute forecast error lies around $2 \mathrm{~K}$ for temperature, $10 \%$ for relative humidity, $1 \mathrm{hPa}$ for pressure, and $1.5 \mathrm{~m} \mathrm{~s}^{-1}$ for wind speed. These values are relatively large because no surface and upperair data assimilation is used on the LAM side. The effect of stochastic physics on RMSE is generally small (Fig. 4) and mostly not statistically significant. Only the hybrid 

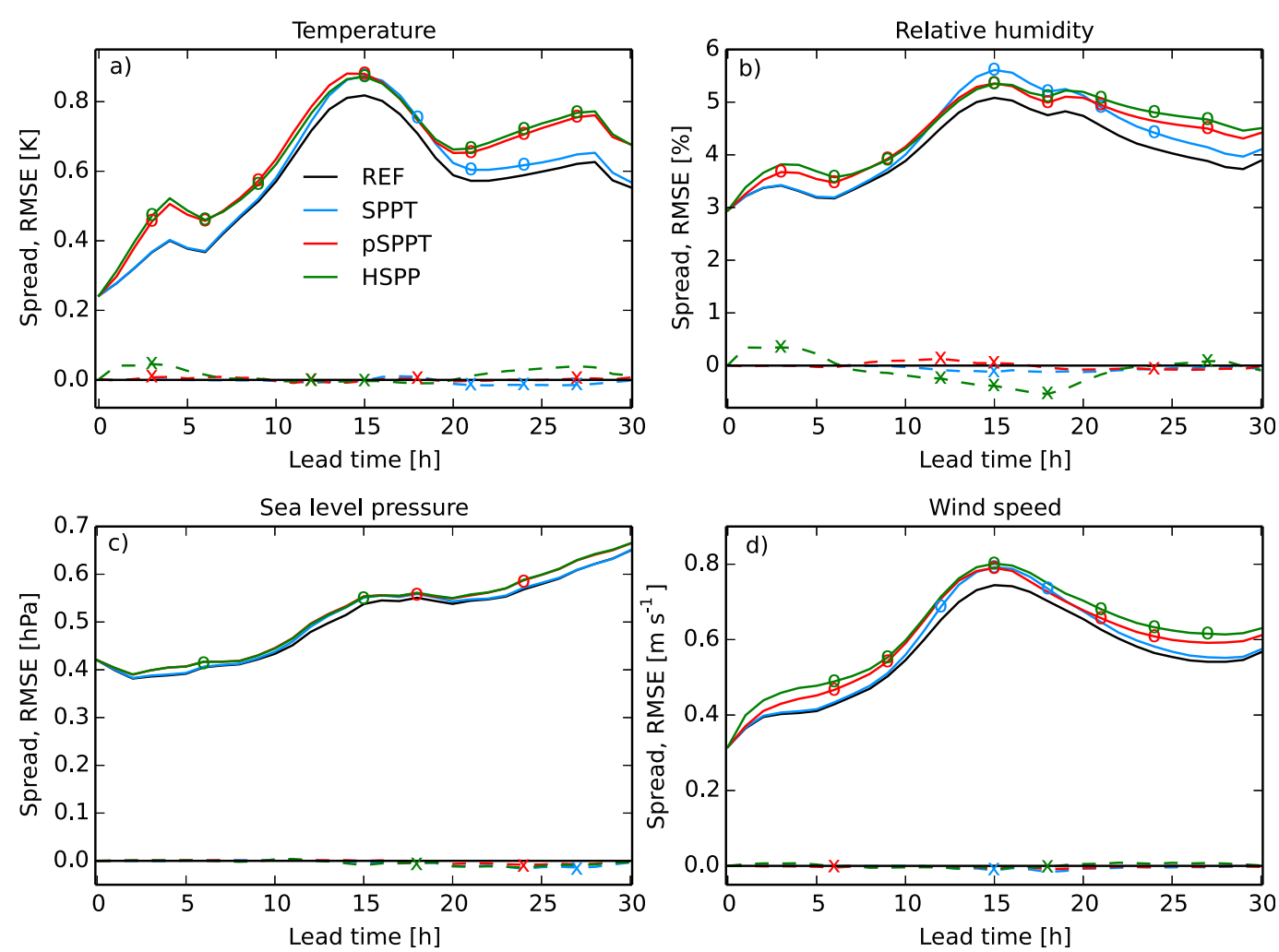

FIG. 4. Ensemble spread (solid lines) and RMSE (dashed lines) as a function of lead time for (a) 2-m temperature, (b) 2-m relative humidity, (c) sea level pressure, and (d) 10-m wind speed in July 2016. RMSE is given as difference to an ensemble without any stochastic physics (REF). Statistically significant differences (shown only every $3 \mathrm{~h}$ ) are indicated by asterisks (RMSE) and circles (spread).

system produces some additional RMSE during the night in the cases of temperature and relative humidity. In the afternoon the forecast error of relative humidity is slightly reduced in HSPP. For sea level pressure and wind speed no clear effect on the RMSE score is obvious.

The greatest ensemble spread in the winter season can be observed for all variables in the HSPP experiment, followed by pSPPT (not shown). The spread of SPPT is similar to the reference experiment. The effect on RMSE is negligible in winter, except for temperature and relative humidity in the HSPP experiment where RMSE is slightly increased. The impact on these scores is generally low in the winter period, which is due to very stable conditions and comparably small uncertainties in the forecasts (W19). The performance of the different ensembles in the winter season is also assessed by the continuous ranked probability score (CRPS) in Fig. 5. The CRPS (Wilks 2011) measures the difference between the forecasted and observed cumulative distribution function. The CRPS can be decomposed into a reliability part that is closely connected to the rank histogram, and a resolution part that is related to the average spread of an ensemble and the behavior of its outliers. Smaller CRPS values are indicating a better forecast. All values shown in Fig. 5 are differences relative to the reference run (experiment - REF)-statistically significant differences are denoted by circles (shown only every $3 \mathrm{~h}$ ). The perturbation of model tendencies in SPPT (blue) and pSPPT (red) has a positive effect on the ensemble performance for all parameters shown here, with the pSPPT approach having a better performance, which is also visible in an increased forecast reliability (not shown). The hybrid system however, displays mixed results between the parameters. For wind speed the use of this hybrid system displays a generally good performance, while for temperature at most lead times a positive CRPS difference to the REF experiment is obvious. This is also reflected in the RMSE (not shown), which is slightly increased in the HSPP experiment for temperature and humidity during the first hours. The fact that this increased RMSE (present also in the summer season; Fig. 4) exists only in the HSPP experiment indicates a problem in the parameter perturbation scheme in the turbulence parameterization. We assume that the amplitude of the perturbations is maybe too high for some parameters, but this needs to be investigated in more detail. 

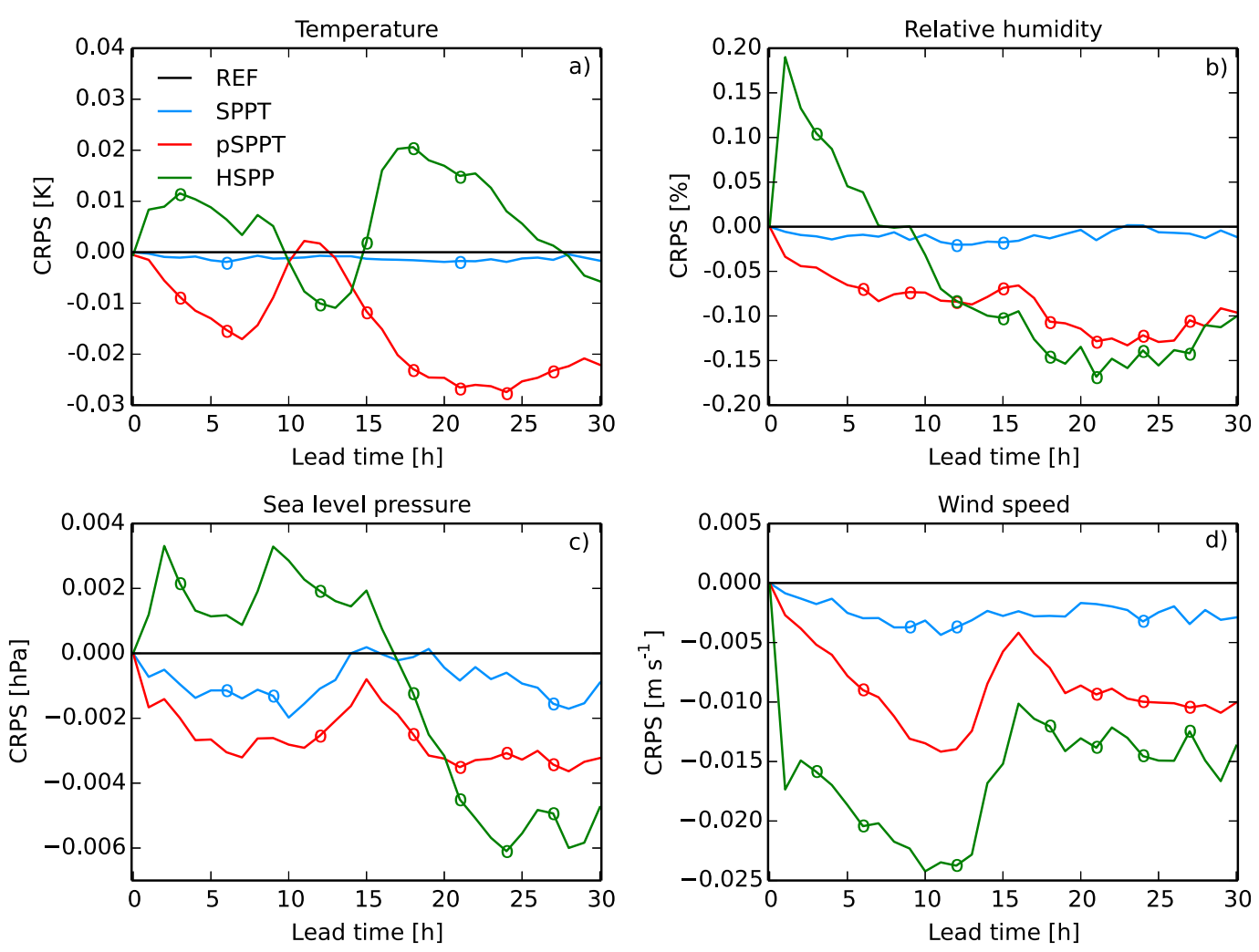

FIG. 5. CRPS as a function of lead time for (a) 2-m temperature, (b) 2-m relative humidity, (c) sea level pressure, and (d) 10-m wind speed in January 2017. Numbers are given as differences to the reference run (REF). Statistically significant differences (shown only every $3 \mathrm{~h}$ ) are indicated by circles.

\section{c. Gridded precipitation verification}

In the case of precipitation the verification is done on the $1 \mathrm{~km}$ INCA grid (Fig. 1). A spatial verification is needed because in such complex terrain as the Alps, precipitation variability is high and a station network alone is insufficient for a representative verification. Accordingly, the INCA analyses are useful in that they also contain precipitation information gained from the radar network (Haiden et al. 2011).

Figure 6 shows the Brier skill score (BSS; Wilks 2011) of the three experiments with stochastic physics depicted for the July 2016 (Figs. 6a,c) and January 2017 (Figs. 6b,d) period. This skill score is used to assess the relative improvement of the experiments (expe) over the reference (ref) run. It is calculated by averaging the Brier score (BS) at each grid point over the test period and applying Eq. (2) afterward:

$$
\mathrm{BSS}(\text { expe })=1-\frac{\mathrm{BS}(\text { expe })}{\mathrm{BS}(\text { ref })} \text {. }
$$

A positive value indicates an improved skill of the experiment compared to the reference without any stochastic physics; 0.3 and $5.0 \mathrm{~mm} \mathrm{~h}^{-1}$ have been chosen as thresholds for the calculation of the Brier score for light and moderate precipitation. $0.3 \mathrm{~mm} \mathrm{~h}^{-1}$ was selected to remove some noise in the INCA analyses from very light precipitation events below this threshold. Statistical significance of the score is indicated by circles in Fig. 6 (only every $3 \mathrm{~h}$ ).

All three experiments with stochastic physics yield a positive BSS for almost all lead times and both precipitation types in summer and winter, respectively. In summer the hybrid system produces the best results, especially for moderate precipitation and lead times between 10 and $20 \mathrm{~h}$. This is the time of the day when most thunderstorm activity is present and where forecasters are faced with the biggest challenges. The situation is similar for light precipitation, although here also the original SPPT approach shows some additional skill, especially in the evening and night hours.

The Brier skill scores for the wintertime period in January 2017 show a different picture. The skill of the forecasts is also generally improved by using stochastic physics, but the ranking among the experiments is not clear. For light precipitation the pSPPT approach performs best, while in case of moderate precipitation the hybrid system outperforms the others for most lead 

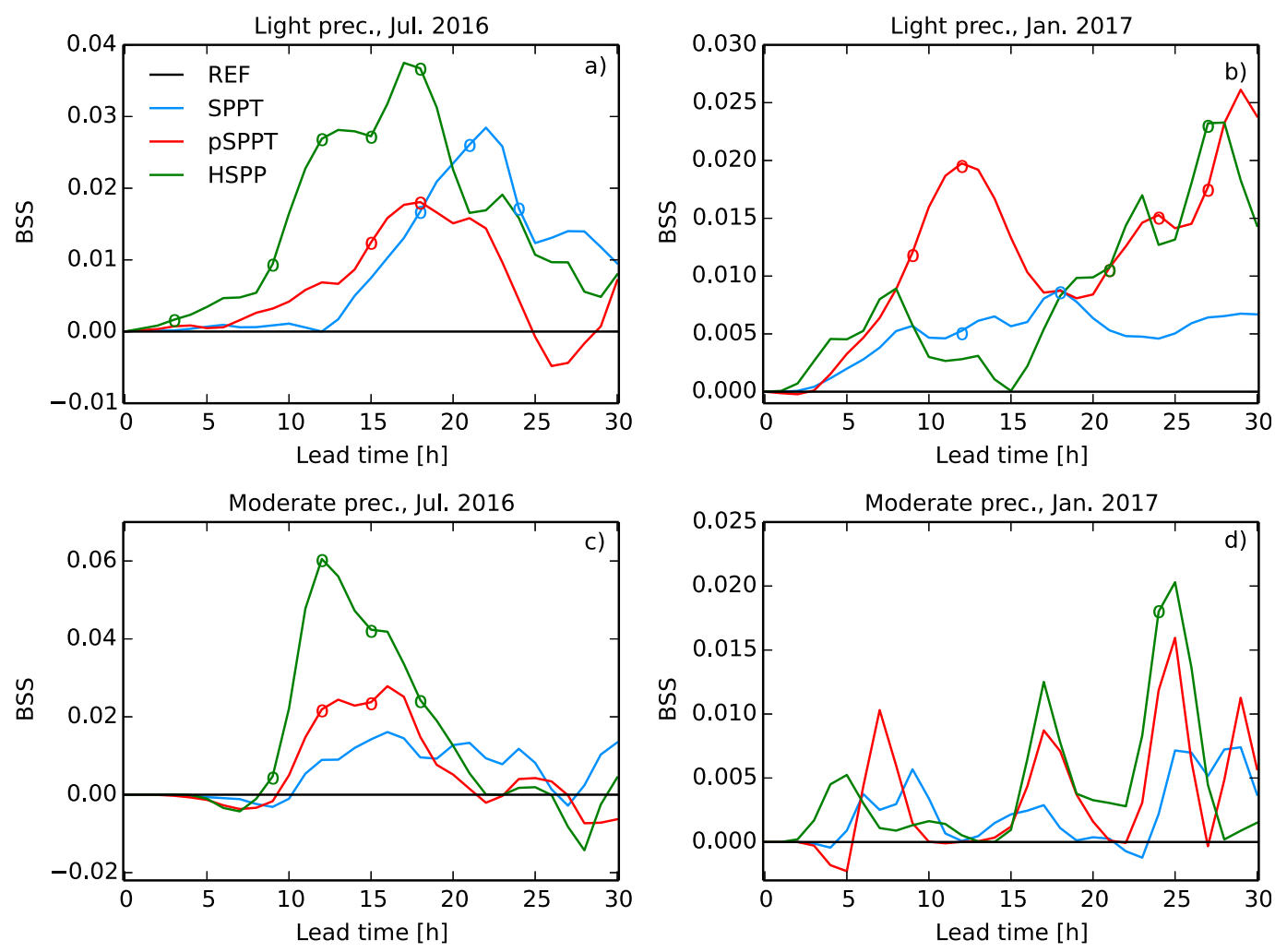

FIG. 6. Brier skill score as a function of lead time for (a),(b) light $\left(0.3-5.0 \mathrm{~mm} \mathrm{~h}^{-1}\right)$ and (c),(d) moderate precipitation $\left(>5.0 \mathrm{~mm} \mathrm{~h}^{-1}\right.$ ) in (a),(c) July 2016 and (b),(d) January 2017, respectively. The skill score is calculated with respect to the reference run (REF). Circles (shown only every $3 \mathrm{~h}$ ) indicate statistically significant scores.

times. However, it has to be pointed out that the effect of stochastic physics on BSS is generally small and not statistically significant in winter, which can be explained by the more stable stratification of the atmosphere in winter (W19). Moreover, the number of moderate precipitation events exceeding the $5.0 \mathrm{~mm} \mathrm{~h}^{-1}$ threshold is rather small in winter. In winter, precipitation events mostly have a large-scale spatial extent with moderate precipitation intensities. Only in areas with strong orographic precipitation enhancement in the Alps this threshold was exceeded regularly. Nevertheless, to be consistent with the summer test period the $5.0 \mathrm{~mm} \mathrm{~h}^{-1}$ threshold is maintained here.

\section{d. Case study with strong convection}

Section $3 \mathrm{c}$ showed that stochastic physics can significantly improve monthly averaged verification scores for precipitation. To further investigate this behavior, a case study with exceptionally high convective activity is selected from the summer test month-25 July 2016. In the afternoon and evening of this day, several intensive thunderstorms developed in southern Germany and in the Alpine area causing heavy precipitation and local floods. Figure 7 a shows the observed $24 \mathrm{~h}$ precipitation sum, Fig. $7 \mathrm{~b}$ shows the areas with observed $24 \mathrm{~h}$ precipitation higher than $20 \mathrm{~mm}$ (in red), and Figs. 7c-f show the probabilities of precipitation higher than $20 \mathrm{~mm}$ in the different experiments. Looking on the reference ensemble (Fig. 7c) indicates a quite good performance of the C-LAEF ensemble in most parts of the domain. However, C-LAEF fails to produce heavy precipitation cells in the Alpine foreland in southern Germany $\left(48^{\circ} \mathrm{N}\right.$, $10^{\circ}-12^{\circ} \mathrm{E}$ ) and overestimates precipitation amounts in the western parts of Austria. The performance of SPPT (Fig. 7d) is comparable to REF, pSPPT (Fig. 7e) shows some improvements (less precipitation) in the southeastern part of the domain and the HSPP approach shows the best performance in southern Germany. Also in southern Austria $\left(46^{\circ}-47^{\circ} \mathrm{N}, 12^{\circ}-14^{\circ} \mathrm{E}\right)$ the precipitation field is best represented in the HSPP experiment, while the other experiments are underestimating precipitation here. The areas with precipitation higher than $20 \mathrm{~mm}$ in $24 \mathrm{~h}$ are generally larger in HSPP than in the other experiments. This originates from a higher spread in HSPP and is closer to the observed precipitation field (Figs. 7a,b).

Test runs with altered amplitudes of the parameter perturbations in HSPP (not shown) revealed a very 

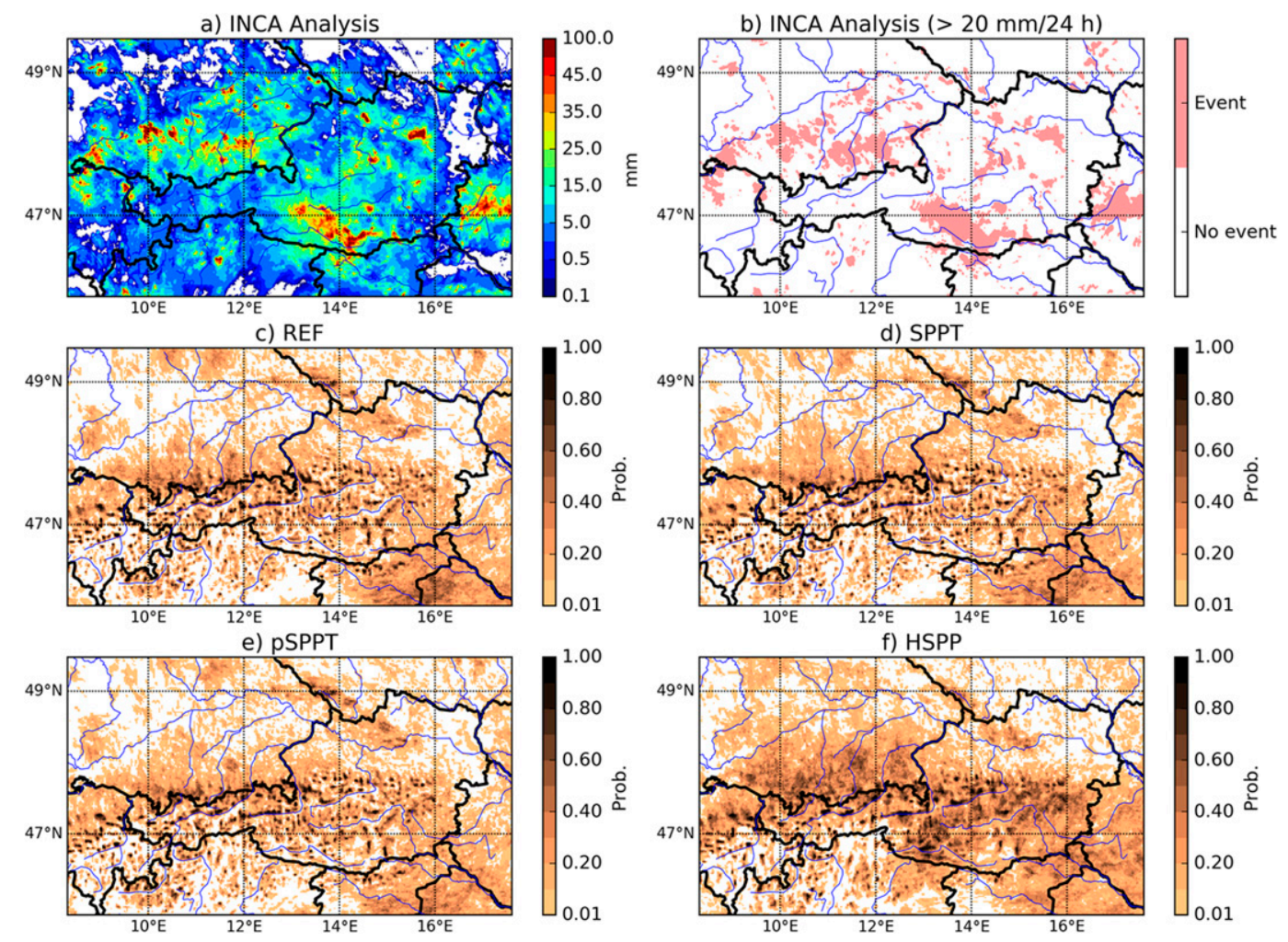

FIG. 7. (a) Observed 24-h INCA precipitation sum for the case study on 25 Jul 2016, (b) areas with observed precipitation higher than $20 \mathrm{~mm}$ in $24 \mathrm{~h}$ (in red), and (c)-(f) respective probabilities of predicted precipitation $>20 \mathrm{~mm}$ in $24 \mathrm{~h}$ in the different C-LAEF experiments [(c) REF, (d) SPPT, (e) pSPPT, and (f) HSPP].

sensitive response of the turbulence scheme and relatively strong effects on the precipitation fields. Some more tuning of the parameters in Table 2 will be necessary to take full advantage of the parameter perturbation scheme. However, it has to be stressed at this point that no data assimilation is used in all experiments shown in Fig. 7, hence some clear improvements can be expected also in this direction.

\section{Discussion and conclusions}

In this study we have proposed a new stochastic physics method that combines tendency perturbations in the radiation, shallow convection and microphysics scheme of C-LAEF and process-based parameter perturbations in the turbulence scheme. The performance of the new hybrid system (HSPP) has been assessed by comparing it with a reference experiment without stochastic physics (REF), one SPPT experiment (Palmer et al. 2009) and one experiment containing an improved version of the pSPPT approach (W19). Results are based on an extensive verification over a summer and a winter month.

The proposed hybrid system combines the strength of a tendency perturbation approach (e.g., simplicity, strong effect) and a parameter perturbation scheme (e.g., conservation of energy). This system is able to solve several inherent problems of the famous SPPT approach:

- A more physical consistent relationship between the different parameterization schemes is sustained due to the interaction among the different schemes.

- The perturbation scale is separately adapted to the uncertainty scale of each physics parameterization.

- Conservation laws are respected. The main criticism to SPPT (Leutbecher et al. 2017) is the lack of energy conservation since only tropospheric model tendencies are perturbed but not fluxes at the surface or at the top of the atmosphere. This causes numerical instabilities and a tapering function has to be introduced to SPPT that smoothly reduces the perturbations to zero in these areas. This problem is circumvented in the proposed hybrid system because uncertainties in the turbulence scheme, which is mainly responsible for the vertical transport in the lower levels, are considered by directly perturbing parameters at the process level. In doing so, a tapering function is not needed in HSPP. HSPP has been developed for the convection-permitting 
C-LAEF ensemble, but in principal this method is applicable to all kind of ensemble forecasting systems (global, regional, non-convection-allowing, etc.).

Generally, the use of stochastic physics leads to improvements in the verification scores of both periods, July 2016 and January 2017. Because of a more stable stratification of the atmosphere in winter, the effect on the scores is generally larger in summer when convective activity is high.

An investigation at 13 vertical levels between the surface and $100 \mathrm{hPa}$ has clearly shown the influence of the tapering function. Switching off tapering significantly increases the ensemble spread in the lower atmosphere, especially for temperature. The effect on specific humidity is smaller because here the treatment of supersaturation plays an important role. Perturbing humidity tendencies (to a smaller extent also temperature tendencies) can easily result in supersaturation. A very simple supersaturation treatment is applied in SPPT, which leads to a negative humidity bias (Bouttier et al. 2012). However, the pSPPT and HSPP experiments use an improved supersaturation adjustment proposed by Szücs (2016). This minimizes the negative humidity bias, but on the other hand also slightly reduces the ensemble spread. The impact of stochastic physics on the forecast error of C-LAEF is generally low and mostly not statistically significant.

From studies with global ensemble systems we would usually expect an increase of ensemble spread with lead time (e.g., Ollinaho et al. 2017). However, the present study reveals a clear diurnal cycle of ensemble spread in summer for most surface parameters. The highest values can be observed in the afternoon and evening hours when convection is present. As convection dissipates during the night the ensemble spread also decreases. This behavior is present in other convection-permitting models as well and has been described e.g., in Nielsen and Schumacher (2016). The July 2016 period was used to investigate this feature by looking on the spatial distribution of ensemble spread in the C-LAEF domain (not shown). The highest mean ensemble spread of precipitation can be found in the afternoon over the Alps and south of it where the highest thunderstorm activity is present. In the geographically flat areas in the north and east of the domain the spread is much lower.

The pSPPT approach presented here and used also in the HSPP is an enhanced version of the one proposed in W19. In addition to an improved supersaturation adjustment, it also contains some modifications in the stochastic pattern itself. In both versions the tendencies of the individual physics parameterization schemes are perturbed separately, but in W19 the perturbation fields differ only in their seed. In the present version the horizontal and temporal scale of the perturbations is also adapted individually to the single parameterizations. This is performed by running the spectral pattern generator separately for each physics parameterization with different settings. The scale settings (Table 1) are defined more or less on personal assessment, providing the smallest perturbations to the scheme with the smallest uncertainty scale (microphysics). The shallow convection scheme is perturbed with slightly largerscale patterns while for radiation the coarsest perturbations are applied (Fig. 2). This simple approach was used because the real uncertainty scale of the different physics parameterization schemes in AROME is not really known. The very few existing studies on uncertainty scales (e.g., Shutts and Pallares 2014) are addressed on larger scale and global models.

A case study with high thunderstorm activity in July 2016 revealed good potential of the HSPP scheme in predicting summertime convection, but it also showed a very sensitive response of the turbulence scheme to variations in the key parameters listed in Table 2. Some more work has to be spent on effectively tuning the amplitude and spatial scales of the perturbations in order to improve probabilistic performance without an error deterioration (as seen in Fig. 5). However, the perturbation of parameters offers a very interesting and promising way of model error representation. In the present hybrid system parameter perturbations are only applied in the turbulence scheme because most problems occurred here when using tendency perturbations. It would be worth-trying to expand this process-based approach to other physics parameterization schemes like shallow convection or microphysics as well. This has already been accomplished for the ECMWF ensemble by Ollinaho et al. (2017) where 20 parameters in all kind of physics parameterizations are perturbed. This SPP scheme displays a slightly lower skill for upper-air variables in the medium range than the SPPT scheme but in the short range the performance is similar. The SPP scheme has the advantage that a tapering function is not needed, and from a physical point of view it is much more consistent to apply perturbations directly at the process level.

Another interesting point considering the stochastic pattern would be a kind of flow dependent pattern generator that creates noise only in areas with high uncertainties. At the moment the perturbation field is created randomly without any consideration of the weather or flow situation. Accordingly, it occurs that strong perturbations are applied in very stable areas (e.g., high pressure areas) without any effect, while on the other hand perturbations are not added in sensible 
areas with strong convection or near frontal zones. Hence it would be much more reasonable to restrict the perturbations to areas with large uncertainties. From a technical point of view this could be achieved during model integration by using information about the vertical stability or the mass flux obtained from a previous time step. However, this would require a complete reconstruction of the stochastic pattern generator and is left for future work. The next step in the development of the C-LAEF system is to combine the model error representations presented here with perturbations of initial and boundary conditions.

Acknowledgments. The authors gratefully thank all of the colleagues who contributed to this study. Special thanks go to Eric Bazile and Yann Seity from MétéoFrance for their input into this work, especially for their help in the selection of the parameters in the turbulence scheme. Furthermore, we want to thank the ECMWF for the possibility to run all the experiments on their supercomputer.

\section{REFERENCES}

Baker, L., A. Rudd, S. Migliorini, and R. Bannister, 2014: Representation of model error in a convective-scale ensemble prediction system. Nonlinear Processes Geophys., 21, 19-39, https://doi.org/10.5194/npg-21-19-2014.

Bénard, P., J. Vivoda, J. Mašek, P. Smolıková, K. Yessad, C. Smith, R. Brožková, and J. F. Geleyn, 2010: Dynamical kernel of the Aladin-NH spectral limited-area model: Revised formulation and sensitivity experiments. Quart. J. Roy. Meteor. Soc., 136, 155-169, https://doi.org/10.1002/qj.522.

Bengtsson, L., M. Steinheimer, P. Bechtold, and J. F. Geleyn, 2013: A stochastic parametrization for deep convection using cellular automata. Quart. J. Roy. Meteor. Soc., 139, 1533-1543, https://doi.org/10.1002/qj.2108.

—_, and Coauthors, 2017: The HARMONIE-AROME model configuration in the ALADIN-HIRLAM NWP system. Mon. Wea. Rev., 145, 1919-1935, https://doi.org/10.1175/MWR-D16-0417.1.

Berner, J., G. J. Shutts, M. Leutbecher, and T. N. Palmer, 2009: A spectral stochastic kinetic energy backscatter scheme and its impact on flow dependent predictability in the ECMWF ensemble prediction system. J. Atmos. Sci., 66, 603-626, https:// doi.org/10.1175/2008JAS2677.1.

Bougeault, P., and P. Lacarrere, 1989: Parameterization of orography-induced turbulence in a mesobeta-scale model. Mon. Wea. Rev., 117, 1872-1890, https://doi.org/10.1175/15200493(1989)117<1872:POOITI>2.0.CO;2.

Bouttier, F., B. Vié, O. Nuissier, and L. Raynaud, 2012: Impact of stochastic physics in a convection-permitting ensemble. Mon. Wea. Rev., 140, 3706-3721, https://doi.org/10.1175/MWR-D12-00031.1.

Bowler, N. E., A. Arribas, K. R. Mylne, K. B. Robertson, and S. E. Beare, 2008: The MOGREPS short-range ensemble prediction system. Quart. J. Roy. Meteor. Soc., 134, 703-722, https://doi.org/10.1002/qj.234.
Buizza, R., M. Miller, and T. N. Palmer, 1999: Stochastic representation of model uncertainties in the ECMWF ensemble prediction system. Quart. J. Roy. Meteor. Soc., 125, 2887-2908, https://doi.org/10.1002/qj.49712556006.

Charron, M., G. Pellerin, L. Spacek, P. L. Houtekamer, N. Gagnon, H. L. Mitchell, and L. Michelin, 2010: Toward random sampling of model error in the Canadian ensemble prediction system. Mon. Wea. Rev., 138,1877-1901, https://doi.org/10.1175/ 2009MWR3187.1.

Christensen, H. M., I. M. Moroz, and T. N. Palmer, 2015: Stochastic and perturbed parameter representations of model uncertainty in convection parameterization. J. Atmos. Sci., 72, 25252544, https://doi.org/10.1175/JAS-D-14-0250.1.

—_, S.-J. Lock, I. M. Moroz, and T. N. Palmer, 2017: Introducing independent patterns into the Stochastically Perturbed Parametrization Tendencies (SPPT) scheme. Quart. J. Roy. Meteor. Soc., 143, 2168-2181, https://doi.org/ 10.1002/qj.3075.

Davies, H., 1976: A lateral boundary formulation for multi-level prediction models. Quart. J. Roy. Meteor. Soc., 102, 405-418, https://doi.org/10.1002/qj.49710243210.

Hacker, J. P., C. Snyder, S. Y. Ha, and M. Pocernich, 2011: Linear and non-linear response to parameter variations in a mesoscale model. Tellus, 63A, 429-444, https://doi.org/10.1111/ j.1600-0870.2010.00505.x.

Haiden, T., A. Kann, C. Wittmann, G. Pistotnik, B. Bica, and C. Gruber, 2011: The Integrated Nowcasting through Comprehensive Analysis (INCA) system and its validation over the eastern alpine region. Wea. Forecasting, 26, 166-183, https:// doi.org/10.1175/2010WAF2222451.1.

Iversen, T., A. Deckmyn, C. Santos, K. Sattler, J. B. Bremnes, H. Feddersen, and I. Frogner, 2011: Evaluation of 'GLAMEPS'A proposed multimodel EPS for short range forecasting. Tellus, 63A, 513-530, https://doi.org/10.1111/j.1600-0870.2010.00507.x.

Jankov, I., and J. Beck, 2019: Stochastically perturbed parameterizations in an HRRR-based ensemble. Mon. Wea. Rev., 147, 153-173, https://doi.org/10.1175/MWR-D-18-0092.1.

Kober, K., and G. C. Craig, 2016: Physically based stochastic perturbations (PSP) in the boundary layer to represent uncertainty in convective initiation. J. Atmos. Sci., 73, 2893-2911, https://doi.org/10.1175/JAS-D-15-0144.1.

Leutbecher, M., and Coauthors, 2017: Stochastic representations of model uncertainties at ECMWF: State of the art and future vision. Quart. J. Roy. Meteor. Soc., 143, 2315-2339, https:// doi.org/10.1002/qj.3094.

Mascart, P. J., and P. Bougeault, 2011: The Meso-NH atmospheric simulation system: Scientific documentation, Part III: Physics. Tech. Rep., Meteo-France, 153 pp., http://mesonh.aero.obsmip.fr/mesonh/dir_doc/book1_m48_19jan2009/scidoc_p3.pdf.

Nielsen, E. R., and R. S. Schumacher, 2016: Using convectionallowing ensembles to understand the predictability of an extreme rainfall event. Mon. Wea. Rev., 144, 3651-3676, https://doi.org/10.1175/MWR-D-16-0083.1.

Ollinaho, P., and Coauthors, 2017: Towards process-level representation of model uncertainties: Stochastically perturbed parametrisations in the ECMWF ensemble. Quart. J. Roy. Meteor. Soc., 143, 408-422, https://doi.org/10.1002/qj.2931.

Palmer, T. N., R. Buizza, F. Doblas-Reyes, T. Jung, M. Leutbecher, G. J. Shutts, M. Steinheimer, and A. Weisheimer, 2009: Stochastic parametrization and model uncertainty. ECMWF Tech. Memo. 598, 44 pp., https://www.ecmwf.int/sites/default/files/ elibrary/2009/11577-stochastic-parametrization-and-modeluncertainty.pdf. 
Plant, R. S., and G. C. Craig, 2008: A stochastic parameterization for deep convection based on equilibrium statistics. J. Atmos. Sci., 65, 87-104, https://doi.org/10.1175/2007JAS2263.1.

Romine, G. S., C. S. Schwartz, J. Berner, K. R. Fossell, C. Snyder, J. L. Anderson, and M. L. Weisman, 2014: Representing forecast error in a convection-permitting ensemble system. Mon. Wea. Rev., 142, 4519-4541, https://doi.org/10.1175/ MWR-D-14-00100.1.

Seity, Y., P. Brousseau, S. Malardel, G. Hello, P. Bénard, F. Bouttier, C. Lac, and V. Masson, 2011: The AROMEFrance convective-scale operational model. Mon. Wea. Rev., 139, 976-991, https://doi.org/10.1175/2010MWR3425.1.

Shutts, G. J., and A. C. Pallares, 2014: Assessing parametrization uncertainty associated with horizontal resolution in numerical weather prediction models. Philos. Trans. Roy. Soc. London, A372, 20130284, https://doi.org/10.1098/rsta.2013.0284.

Szücs, M., 2016: SPPT in AROME and ALARO. HIRLAM Working Week on EPS and Predictability 2016-2, Helsinki, Finland, ALADIN, 35 pp.

Termonia, P., and Coauthors, 2018: The ALADIN system and its canonical model configurations AROME CY41T1 and ALARO CY40T1. Geosci. Model Dev., 11, 257-281, https://doi.org/ 10.5194/gmd-11-257-2018.
Wang, Y., and Coauthors, 2011: The Central European limited-area ensemble forecasting system: ALADIN-LAEF. Quart. J. Roy. Meteor. Soc., 137, 483-502, https://doi.org/10.1002/qj.751.

_ management and risk prevention in a transnational and interdisciplinary framework. Meteor. Z., 26, 459-473, https:// doi.org/10.1127/metz/2017/0843.

_ _ , and Coauthors, 2018: 27 years of Regional Cooperation for Limited Area Modelling in Central Europe. Bull. Amer. Meteor. Soc., 99, 1415-1432, https://doi.org/10.1175/BAMS-D-160321.1.

Wastl, C., Y. Wang, A. Atencia, and C. Wittmann, 2019: Independent perturbations for physics parametrization tendencies in a convection-permitting ensemble (pSPPT). Geosci. Model Dev., 12, 261-273, https://doi.org/10.5194/gmd-12-2612019.

Weisheimer, A., S. Corti, T. N. Palmer, and F. Vitart, 2014: Addressing model error through atmospheric stochastic physical parametrizations: Impact on the coupled ECMWF seasonal forecasting system. Philos. Trans. Roy. Soc. London, A372, 20130290, https://doi.org/10.1098/rsta.2013.0290.

Wilks, D. S., 2011: Statistical Methods in the Atmospheric Sciences. 3rd ed. International Geophysics Series, Vol. 100, Academic Press, 704 pp. 